\title{
A Novel Mutation in FOXF1 Gene Associated with a Delayed Presentation of Alveolar Capillary Dysplasia with Misalignment of Pulmonary Veins
}

\author{
Maxine S. Eikani' ${ }^{1}$, Vladimir V. Kalinichenko², Arun Pradhan², Julie Noe1 \\ ${ }^{1}$ Department of Pediatric Pulmonary and Sleep Medicine, Medical College of Wisconsin, Milwaukee, USA \\ ${ }^{2}$ Division of Pulmonary Biology, Cincinnati Children's Hospital Medical Center, Cincinnati, USA \\ Email: $\underline{\text { meikani@mcw.edu }}$
}

Received 28 February 2015; accepted 14 March 2015; published 18 March 2015

Copyright () 2015 by authors and Scientific Research Publishing Inc.

This work is licensed under the Creative Commons Attribution International License (CC BY).

http://creativecommons.org/licenses/by/4.0/

(c) () 0 pen Access

\begin{abstract}
Alveolar capillary dysplasia with misalignment of pulmonary veins (ACD/MPV) is a rare, fatal developmental lung disorder of neonates and infants, associated with severe persistent pulmonary hypertension unresponsive to treatment. We reported the case of a term newborn with delayed presentation of ACD/MPV and a novel mutation of FOXF1 gene that received supportive cardiopulmonary treatments, inhaled nitric oxide, oral sildenafil and nebulized iloprost with no clinical improvement. DNA sequence analysis of FOXF1 gene identified a novel heterozygous variant c.257G > C; p.R86P, in exon 1. At autopsy, lung histology showed the characteristic features of ACD/MPV. FOXF1 has been identified as one of the genes responsible for ACD/MPV associated with multiple congenital malformations. This is a report of a novel heterozygous variant c.257G > C; p.R86P, in the first exon of FOXF1, in a patient with delayed presentation of ACD/MPV.
\end{abstract}

\section{Keywords}

Alveolar Capillary Dysplasia with Misalignment of Pulmonary Veins, Persistent Pulmonary Hypertension, FOXF1

\section{Introduction}

Alveolar capillary dysplasia with misalignment of pulmonary veins (ACD/MPV) is a fatal pulmonary disease that usually presents in the neonatal period with severe hypoxemia and persistent pulmonary hypertension un- 
responsive to treatment. The disorder is mostly diagnosed at autopsy or lung biopsy [1]. ACD/MPV is a rare disorder with an unknown incidence and the pathophysiology remains incompletely understood. The genetic etiology of ACD/MPV has remained elusive for decades [1]. Pedigree analyses of ACD/MPV patients' families have suggested both autosomal dominant and recessive patterns of inheritance [2], thus implicating at least two causative genes. Microdeletions or point mutations in/or upstream to the forkhead box F1 (FOXF1) gene on chromosome 16q24.1 are responsible for $40 \%$ of the reported cases [2]. The authors report a clinical case of a term newborn with delayed presentation of ACD/MPV and a novel mutation of FOXF1 gene. Sequence analysis of FOXF1 gene identified a novel heterozygous variant c.257G > C; p.R86P, in exon 1.

\section{Case Report}

A 39-week gestational age Caucasian female was transferred to our center on her sixth day of life for episodes of cyanosis and apnea. The pregnancy was uneventful and no respiratory support was required after birth. She was discharged home from a level one newborn nursery on the second day of life. Parents reported feeding difficulties and few self-limited episodes of perioral cyanosis at home. These episodes progressed leading to evaluation in the Emergency Department. She was admitted to the pediatric intensive care unit for hypoxia and frequent apneic events. Her physical exam on admission was significant for bilateral inferior coloboma and lung exam was unremarkable. Chest radiograph showed right sided pneumothorax requiring chest tube placement (Figure 1(a) and Figure 1(b)). Echocardiography was significant for severe pulmonary hypertension, patent foramen ovale (right-left shunt) and small patent ductus arteriosus (right-left shunt). On day 7 of life, she required intubation, and mechanical ventilation due to respiratory failure and increasing $\mathrm{FiO}_{2}$ requirement. Inhaled nitric oxide was started at the same time. Since there was no evidence of clinical improvement, oral sildenafil and nebulized iloprost were added. On day 9 of life she was placed on venoarterial extracorporeal membrane oxygenation (ECMO). A CT scan of the chest with contrast showed normal pulmonary arteries, asymmetric smooth intralobular septal thickening and bilateral lower lobe atelectasis (Figure 2(a) and Figure 2(b)).

On day 21 of life, sequence analysis of FOXF1 identified a novel heterozygous mutation (c.257G > C; p. R86P) in exon 1. The c.257G > C; p.R86P FOXF1 mutation resulted in a single aminoacid substitution of evolutionary conserved Arginine 86 to Proline (Figure 3(a)). Arginine 86 is located in FOXF1 DNA-binding domain (Figure 3(b)), which is frequently mutated in ACD/MPV patients [3]. This mutation was not detected in patient's parents, suggesting of a de novo variant.

On day 26 of life, ECMO support was discontinued due to a left cerebral hemorrhagic stroke. Patient was extubated and remained on maximal medical management for pulmonary hypertension. She died on day 29 of life from frequent pulmonary hypertensive crises. Limited lungs autopsy was performed revealing characteristic histologic features of ACD/MPV (Figure 4).

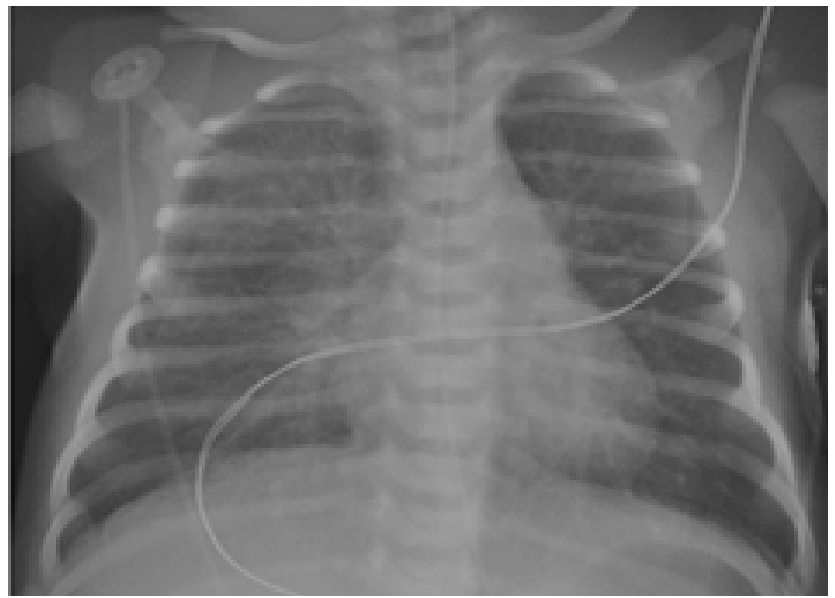

(a)

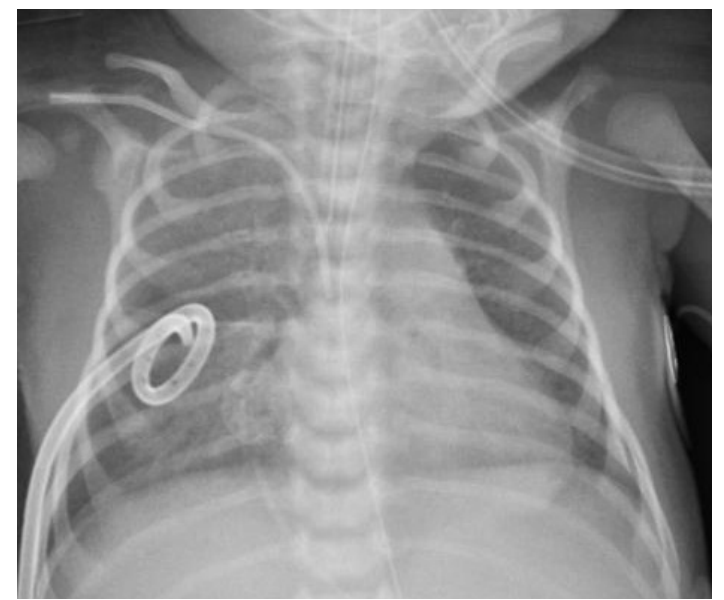

(b)

Figure 1. Chest Radiographs on day of life 7. (a) Slight hazy interstitial opacities throughout both lung fields and small right sided pneumothorax. The cardiothymic silhouette is normal; (b) Chest X-ray after right sided chest tube placement and intubation. 


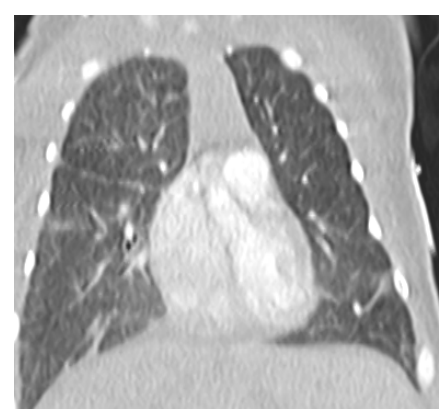

(a)

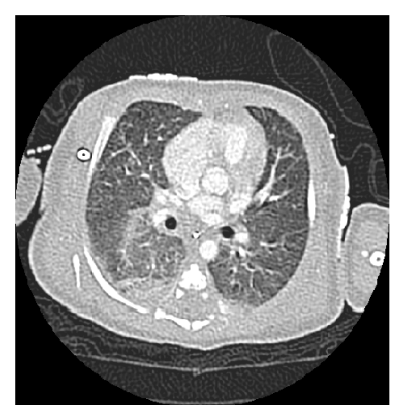

(b)

Figure 2. Chest computed tomography (CT) with contrast on day of life 9. (a) and (b) Normal pulmonary arteries. Smooth intralobular septal thickening which is asymmetric, right greater than left. Bilateral lower lobe atelectasis.

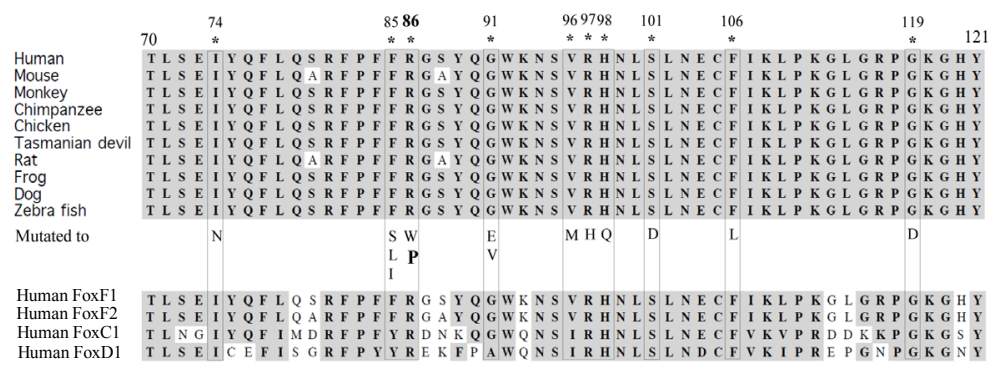

(a)

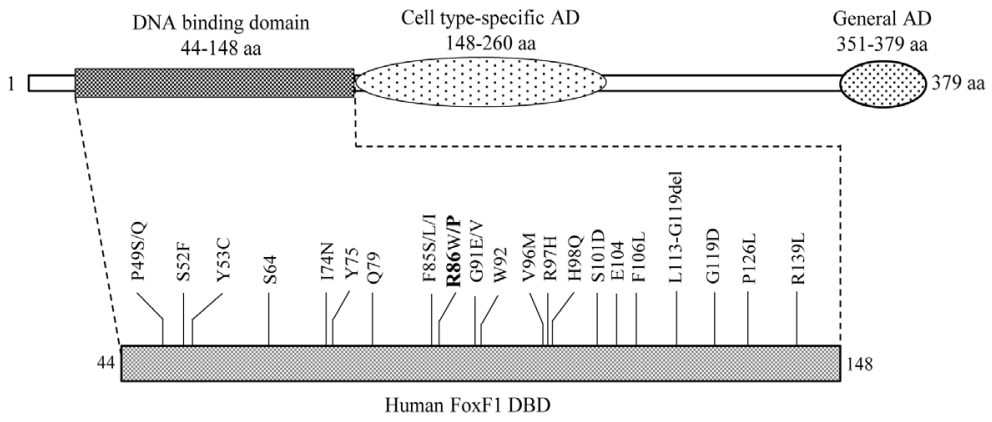

(b)

Figure 3. (a) Multiple sequence alignment displaying the conservation of amino acid sequence (from 70 to 121) of FOXF1 protein among the vertebrates (upper) and between other forkhead box members (lower); (b) Schematic representation of domain organization in human FOXF1 protein (upper) and location of all known mutations in FOXF1 DNA binding domain (lower).

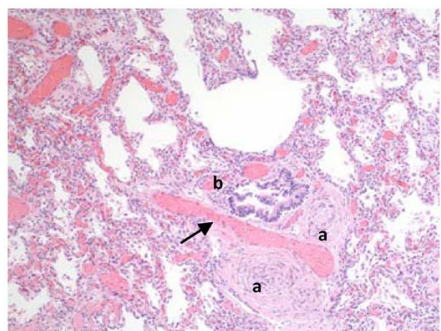

(a)

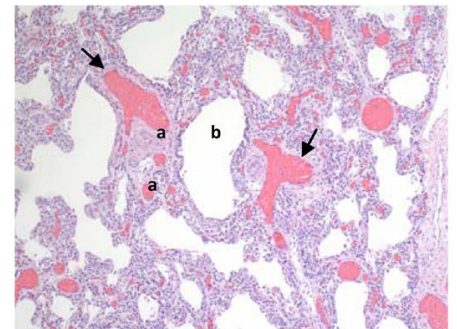

(b)

Figure 4. Autopsy limited to lung biopsy. Confirmatory Diagnosis: Alveolar capillary dysplasia with misalignment of pulmonary veins. (a) and (b) Pulmonary veins (arrows) that are normally in the interlobular septa are malpositioned and accompany pulmonary arteries (a) and bronchioles (b) (H \& E stain). 


\section{Literature Review and Discussion}

$\mathrm{ACD} / \mathrm{MPV}$ is characterized by misaligned pulmonary veins, paucity of capillaries proximal to the alveolar epithelium, anomalous distended pulmonary veins within the bronchovascular bundle instead of the interlobular septa, and immature alveolar development with medial thickening of small pulmonary arteries [1]. It is a developmental disorder of the lung affecting both the parenchyma and the vasculature [2].

Most patients present with respiratory distress in the first few hours of life. These neonates are usually born at term with appropriate size and normal Apgar scores. There are few reports of delayed presentation of this disease. The pathophysiology underlying the development of ACD/MPV has yet to be fully characterized, and mechanisms that might explain delayed presentation are even less clear. There appears to be no survival advantage in presenting late. Once symptoms develop, the infant with delayed presentation experiences the same downward spiral that results in death [4]-[7].

Infants with ACD/MPV usually present with profound hypoxemia and severe pulmonary hypertensive crisis with minimal or no parenchymal lung disease on imaging. It should also be considered in cases of idiopathic persistent pulmonary hypertension of newborn whose symptoms recur after successfully weaning from ECMO [8]. Extrapulmonary findings are present in $50 \%$ to $80 \%$ of the cases which include structural abnormalities of the genitourinary, gastrointestinal, or cardiovascular systems [2] [9] [10].

To date, no routine laboratory tests or imaging studies can distinguish ACD/MPV from other causes of neonatal pulmonary hypertension. Histologic evaluation of lung tissue is the current gold standard for diagnosis and is most often performed at autopsy [10].

It has been reported that FOXF1 plays a crucial role in human lung morphogenesis and intrinsic pulmonary vascular development and thought to be involved in gastrointestinal tract development [11] [12]. FOXF1 is a transcription factor from Forkhead (FOX) family, which is required for embryonic development of pulmonary vasculature in mice and humans by regulating genes critical for the vascular endothelial growth factor (VEGF), platelet-derived growth factor (PDGF) and Notch signaling pathways [13] [14]. Haploinsufficiency of the Foxfl gene in Foxf $1+/-$ mice causes lung hypoplasia, alveolar capillary dysplasia and a variety of developmental abnormalities in the lung and gastrointestinal tract, partially re-capitulating clinical findings in ACD/MPV patients [11]-[14].

DNA sequencing and comparative genomic hybridization have led to the identification of FOXF1 as one of the genes responsible for ACD/MPV. Stankiewicz et al. identified four different de novo heterozygous mutations in the FOXF1 gene in unrelated patients with sporadic ACD/MPV [10] [15]. Subsequently, more than 40 mutations in FOXF1 gene locus were linked to ACD/MPV, including a point mutation c.256C > T; p.R86W, which resulted in a substitution of Arginine 86 to Tryptophan [13]. All FOXF1 mutations are heterozygous, affecting only one FOXF1 allele. Interestingly, the R86W mutation resulted in delayed presentation of the disease, which is similar to the present report of R86P mutation. Therefore, Arginine 86 FOXF1 mutations may have a predictive value for delayed presentation of ACD/MPV. Although overall significance of FOXF1 mutations for cellular functions of FOXF1 protein and pulmonary vascular development remains unclear, our report together with previous data suggest a critical importance of Arginine 86 for pathogenesis of ACD/MPV [3].

\section{Conclusion}

In summary, this is the first report of a heterozygous variant c.257G $>$ C; p.R86P, in the first exon of FOXF1 gene, a critical developmental regulator of pulmonary endothelial cells. The R86P FOXF1 mutation affected only one FOXF1 allele but was sufficient to cause ACD/MPV. While this case was associated with a delay in onset of the disease, it ultimately resulted in progressive respiratory insufficiency refractory to available treatments. Due to the lack of major improvements in ACD/MPV clinical management, new therapeutic approaches are greatly needed for this rare congenital disorder.

\section{Ethics}

Informed consent for reporting patient's history was obtained from the family.

\section{Acknowledgements}

We thank Dr James Southern for his support with the histologic and pathologic findings. 


\section{Conflict of Interest}

The authors declare no conflict of interest.

\section{References}

[1] Bishop, N.B., Stankiewicz, P. and Steinhorn, R.H. (2011) Alveolar Capillary Dysplasia. American Journal of Respiratory and Critical Care Medicine, 184, 172-179. http://dx.doi.org/10.1164/rccm.201010-1697CI

[2] Sen, P., Thakur, N., Stockton, D.W., Langston, C. and Bejjani, B. (2004) Expanding the Phenotype of Alveolar Capillary Dysplasia (ACD). The Journal of Pediatrics, 145, 646-651. http://dx.doi.org/10.1016/j.jpeds.2004.06.081

[3] Sen, P., Yang, Y., Navarro, C., Silva, I., Szafranski, P., Kolodziejska, K.E., et al. (2013) Novel FOXF1 Mutations in Sporadic and Familial Cases of Alveolar Capillary Dysplasia with Misaligned Pulmonary Veins Imply a Role for Its DNA Binding Domain. Human Mutation, 34, 801-811. http://dx.doi.org/10.1002/humu.22313

[4] Ahmed, S., Ackerman, V., Faught, P. and Langston, C. (2008) Profound Hypoxemia and Pulmonary Hypertension in a 7-Month-Old Infant: Late Presentation of Alveolar Capillary Dysplasia. Pediatric Critical Care Medicine, 9, e43-e46. http://dx.doi.org/10.1097/PCC.0b013e31818e383e

[5] Abdallah, H.I., Karmazin, N. and Marks, L.A. (1993) Late Presentation of Misalignment of Lung Vessels with Alveolar Capillary Dysplasia. Critical Care Medicine, 21, 628-630. http://dx.doi.org/10.1097/00003246-199304000-00026

[6] Shankar, V., Haque, A., Johnson, J. and Pietsch, J. (2006) Late Presentation of Alveolar Capillary Dysplasia in an Infant. Pediatric Critical Care Medicine, 7, 177-179. http://dx.doi.org/10.1097/01.PCC.0000202570.58016.67

[7] Shehata, B.M. and Abramowsky, C.R. (2005) Alveolar Capillary Dysplasia in an Infant with Trisomy 21. Pediatric and Developmental Pathology, 8, 696-700. http://dx.doi.org/10.1007/s10024-005-2137-6

[8] Fisher, J.C., Stolar, C.J.H. and Cowles, R.A. (2008) Extracorporeal Membrane Oxygenation for Cardiopulmonary Failure in Pediatric Patients: Is a Second Course Justified? Journal of Surgical Research, 148, 100-108. http://dx.doi.org/10.1016/j.jss.2008.03.023

[9] Antao, B., Samuel, M., Kiely, E., Spitz, L. and Malone, M. (2006) Congenital Alveolar Capillary Dysplasia and Associated Gastrointestinal Anomalies. Fetal \& Pediatric Pathology, 25, 137-145. http://dx.doi.org/10.1080/15513810600908230

[10] Bishop, N., Stankiewicz, P. and Steinhorn, R. (2011) Alveolar Capillary Dysplasia. American Journal of Respiratory and Critical Care Medicine, 184, 172-179. http://dx.doi.org/10.1164/rccm.201010-1697CI

[11] Mahlapuu, M., Enerbäck, S. and Carlsson, P. (2001) Haploinsufficiency of the Forkhead Gene Foxf1, a Target for Sonic Hedgehog Signaling, Causes Lung and Foregut Malformations. Development, 128, 2397-2406.

[12] Kalinichenko, V.V., Gusarova, G.A., Kim, I.M., Shin, B., Yoder, H.M., Clark, J., Sapozhnikov, A.M., Whitsett, J.A. and Costa, R.H. (2004) Foxf1 Haploinsufficiency Reduces Notch-2 Signaling during Mouse Lung Development. American Journal of Physiology-Lung Cellular and Molecular, 286, L521-L530. http://dx.doi.org/10.1152/ajplung.00212.2003

[13] Kalinichenko, V.V., Gusarova, G.A., Shin, B., Costa, R.H. (2003) The Forkhead Box F1 Transcription Factor Is Expressed in Brain and Head Mesenchyme during Mouse Embryonic Development. Gene Expression Patterns, 3, 153158. http://dx.doi.org/10.1016/S1567-133X(03)00010-3

[14] Kalinichenko, V.V., Bhattacharyya, D., Zhou, Y., Gusarova, G.A., Kim, W., Shin, B. and Costa, R.H. (2002) Foxf1

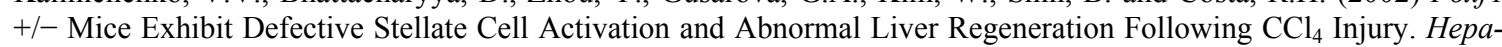
tology, 37, 107-117. http://dx.doi.org/10.1053/jhep.2003.50005

[15] Stankiewicz, P., Sen, P., Bhatt, S., Storer, M., Xia, Z., Bejjani, B.A., et al. (2009) Genomic and Genetic of the FOX Gene Cluster on 16q24.1 and Inactivating Mutations of the FOXF1 Cause Alveolar Capillary Dysplasia and Other Malformations. American Journal of Human Genetics, 84, 780-791. http://dx.doi.org/10.1016/j.ajhg.2009.05.005 